\title{
Comparative Study of Sonourethrography and Radiographic Urethrogaphy in Urethral Lesions
}

\author{
Rauniyar RK ${ }^{1}$, Agrawal CS ${ }^{2}$, Gupta MK ${ }^{1}$, Ahmad K ${ }^{1}$, Gupta A ${ }^{1}$, Baboo S $^{1}$ \\ ${ }^{1}$ Department of Radiodiagnosis and Imaging, ${ }^{2}$ Department of Surgery, BPKIHS, Dharan, \\ Nepal
}

\begin{abstract}
Background: The radiographic urethrography is an invasive, time consuming procedure and due to risk of radiation exposure can't be repeated when required, further it has failed to demonstrate periurethral abnormality as a cause of urethral lesion. Where as sonourethrography is a noninvasive, painless, cheap, quick, easy to perform and without radiation exposure and provide detail visualization of mucosal and periurethral soft tissue.

Objectives: To establish the accuracy of sonourethrography in detecting urethral lesions. Materials and Methods: Study was conducted as single blind prospective study to compare the radiographic urethrography (RGU/MCU) and sonourethragraphy and to established their role and accuracy in evaluation of urethral abnormalities. The findings and results of radiographic urethrography and sonourethrography were correlated and considering radiographic urethrography as gold standard, accuracy of sonourethrography in diagnosis of urethral lesions were analyzed. Result: Total of 27 patients were included in the study. RGU showed normal anterior (penile and bulbar) urethra in 12 out of 27 cases while among these only 9 were normal on sonourethrography. The length of stricture measured on sonourethrography was slightly more than as measured on RGU. Periurethral causes for urethral abnormality were completely identified on sonourethrography while they were not identified on RGU. Conclusion: Radiographic urethrography though gold standard in evaluating urethral abnormalities, sonourethrography is equally accurate (sensitivity 100\%) in the diagnosis of urethral lesions. Sonourethrography has advantage in better evaluating mucosal and periurethral abnormalities as cause of urethral lesions. Further sonourethrography is more accurate in measurement of stricture length and it is cheap, safe and does not provide any kind of radiation to the patients in gonad areas.
\end{abstract}

Keywords: Micturating Cysto-Urethrography, Retrograde Urethrography, Sonourethrography

\section{Introduction}

Radiographic Urethrography either ante grade (Micturating cysto-urethrography MCU or Retrograde Urethrography RGU) is
Corrospondence to: Prof.Dr. R. K. Rauniyar, Professor and Head, Department of Radiodiagnosis and Imaging, BPKIHS, Dharan, Nepal

Email:- rauniyar99@yahoo.com 
the most common investigation in imaging evaluation of the urethra in various urethral lesions. MCU approach is preferred for assessing the abnormality of the prostatic urethra. RGU better depicts the membranous and anterior urethra. Ultrasonography (US) is able to assess both urethral and periurethral tissue. MRI provides excellent soft tissue contrast, depicts the urethra and periurethral tissue to best advantage, but it is expensive and universal availability is a problem. There are several reports available in literature on sonourethrography ${ }^{1-12}$ but there are no clear cut information regarding the exact role of sonourethrography and radiographic urethrography (RGU/MCU) in evaluation of urethral and periurethral abnormalities and further the accuracy of this newer technique over conventional urethrography is not known. Radiographic urethrography has traditionally been the gold standard for evaluation of urethral lesion. The availability of superficial high frequency (7.5-10 MHz) and high resolution ultrasound probe has revolutionized the imaging of urethra. The radiographic urethrography is a invasive, costly, time consuming procedure and due to the risk of radiation exposure can't be repeated when required, further it has failed to demonstrate periurethral abnormality as a cause of urethral lesion. Whereas sonourethrography is a noninvasive, painless, cheap, quick, easy to perform and without radiation exposure and provides detailed visualization of mucosal and periurethral soft tissue. Further it does not use any radiographic contrast material. Hence with this advantage of sonourethrography, this study was conducted to establish the role of sonourethrography and its accuracy in evaluation of urethral lesions.

\section{Material and Method}

This was single blind prospective study conducted in the department of Radiodiagnosis to compare the conventional radiographic urethrography (RGU/MCU) and sonourethrography and to establish their role and accuracy in evaluation of urethral abnormalities. All the patients undergoing ante grade and retrograde urethrography for urethral lesions were included in the study while the patients with urinary tract infection (UTI) and active urethral (urethritis) diseases were excluded.

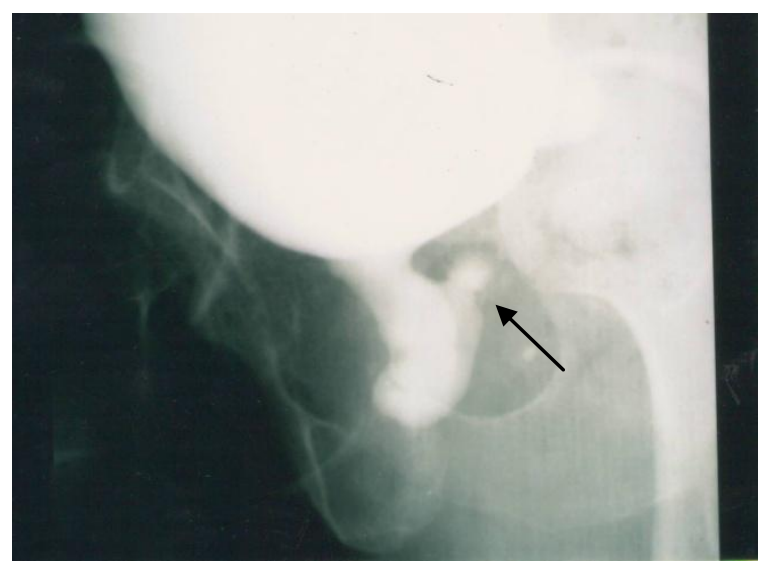

Fig 1a: MCU showing dilated posterior urethra with abrupt cut off with small diverticulum (arrow).

\section{Radiographic Urethrography}

First RGU was performed after taking informed consent under full aseptic precaution. Dilute radiographic contrast material was injected retrogradely by placing tip of the infant feeding tube connected to contrast loaded syringe at just inside external urethral meatus and urethral orifice was occluded to prevent reflux of contrast by compressing the glans penis between two fingers. In few cases urethral occlusion was achieved by Foley's catheter with balloon inflated in the fossa navicularis. While 
contrast was injected radiographs were obtained in both right and left oblique view to profile the urethra. Whenever required RGU was followed by MCU to evaluate entire urethra. For MCU bladder was distended with dilute contrast via either already placed supra-pubic catheter or by retrograde catheterization of infant feeding tube under full aseptic precaution after injecting $2 \%$ lignocaine jelly before catheterization. Right and left oblique views radiographs were obtained while patient was voiding on table.

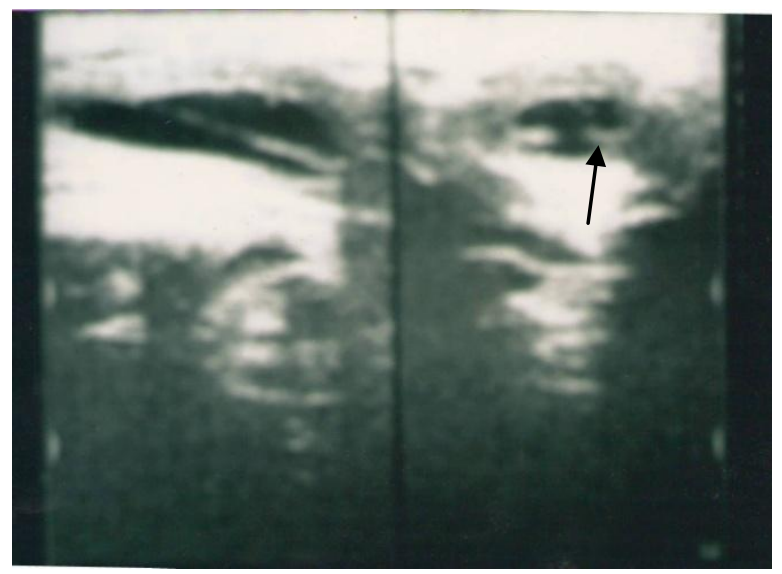

Fig 1b: Sonourethrography of same patients (longitudinal and axial scan) showing transverse fold of membrane suggesting posterior urethral valve.

\section{Sonourethrography}

After obtaining informed consent, all patients underwent sonourethrography under full aseptic precaution on the same day. Sonourethrography were carried out on Sonoline versa (SIEMENS) equipment with 7.5 $\mathrm{MHz}$ linear transducer either by dorsal or ventral aspect of penis after distending the urethra with sterile saline solution. The lumen of the urethra was visualized as anechoic tubular structure when filled with saline and urethral wall as hyper echoic.
On sonourethrography the periurethral tissue were also evaluated after distending the urethra using infant feeding tube connected to preloaded saline syringe with tip of tube placed just inside external meatus which was occluded by compressing glans penis placed between the fingers.

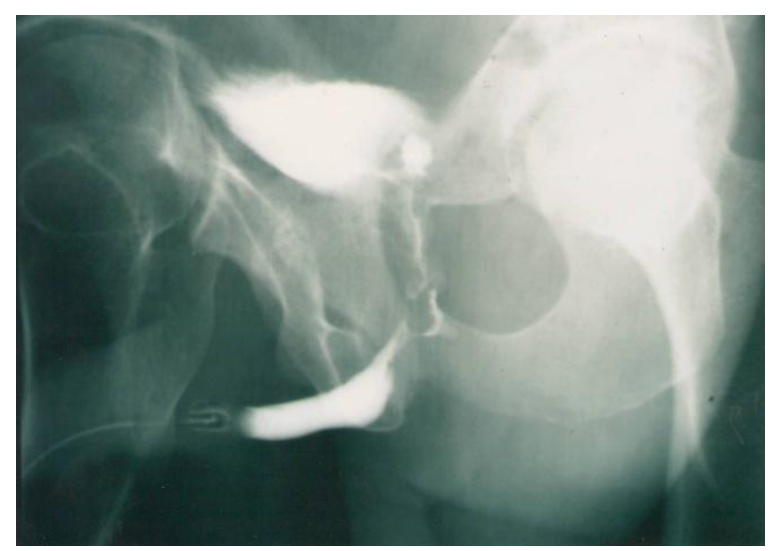

Fig 2a: RGU showing irregular narrowing of posterior part of penile urethra (posterior urethra not distended on RGU)

\section{Results}

Clinical presentation of the patients undergoing RGU/MCU are presented in the table I

\section{Table-I: Clinical presentation $(\mathrm{N}=27)$}

\begin{tabular}{ll}
\hline Clinical presentation & $\%$ \\
\hline Poor Stream & $15(55.5 \%)$ \\
\hline $\begin{array}{l}\text { Near retention } \\
\text { Retention following } \\
\text { trauma }\end{array}$ & $2(7.4 \%)$ \\
$\begin{array}{l}\text { Poor stream following } \\
\text { urethritis }\end{array}$ & $1(25.92 \%)$ \\
$\begin{array}{l}\text { Pain during micturation } \\
\text { Haematuria following } \\
\text { trauma }\end{array}$ & $1(3.7 \%)$ \\
\hline
\end{tabular}

RGU showed normal anterior (penile and bulbar) urethra in 12 out of 27 cases while only 9 were normal on sonouethrography. In 
two cases mucosal irregularities and in one case elevated mucosal flap were noted in anterior Urethra on sonourethrography.

This showed sonourethrography is better modalities in evaluating subtle mucosal and periurethral abnormalities

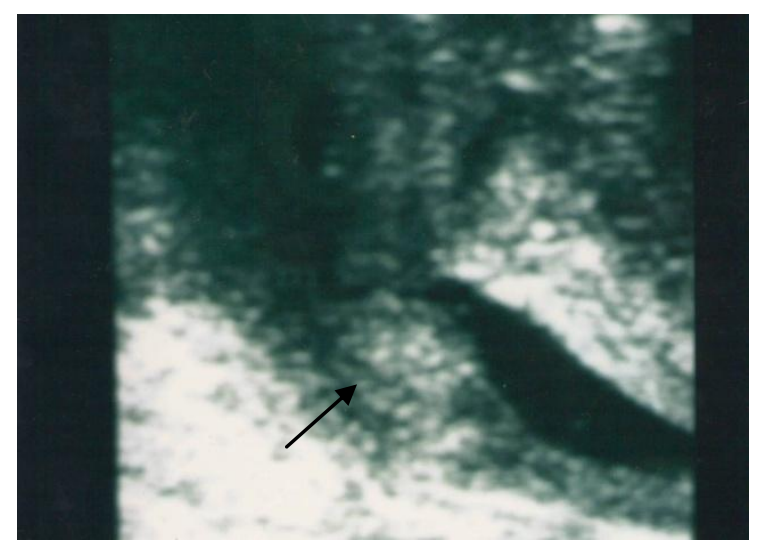

Fig 2b: Sonourethrography showing periurethral echogenic soft tissue lesion suggesting hematoma (in view of trauma) causing narrowing and compression of posterior part of penile urethra.

\section{Urethral Stricture}

All the case of partial and complete stricture on RGU was identified on sonourethrography. There were 7 cases of partial stricture, two cases of complete stricture, two case of narrowed urethra and one case of PUV in the study as presented in table II. The length of stricture measured on sonourethrography was slightly more than as measured on RGU. Measurement on sonourethrography appears to be more accurate than measurement on RGU. Further complete stricture case showed soft tissue at the site of stricture on sonourethrography which were not seen on Radiographic Urethrpgraphy.

Table II-Stricture length on RGU and sonourethrography

\begin{tabular}{lll}
\hline SN & RGU & Sonourethrography \\
\hline $\mathbf{1}$ & $3.3 \mathrm{~cm}$ & $4.0 \mathrm{~cm}$ \\
$\mathbf{2}$ & $6.0 \mathrm{~cm}$ & $7.9 \mathrm{~cm}$ \\
$\mathbf{3}$ & $1.5 \mathrm{~cm}$ & $2.0 \mathrm{~cm}$ \\
$\mathbf{4}$ & $3.0 \mathrm{~cm}$ & $3.8 \mathrm{~cm}$ \\
$\mathbf{5}$ & $4.8 \mathrm{~cm}$ & $5.3 \mathrm{~cm}$ \\
$\mathbf{6}$ & $2.3 \mathrm{~cm}$ & $2.5 \mathrm{~cm}$ \\
$\mathbf{7}$ & $1.3 \mathrm{~cm}$ & $1.6 \mathrm{~cm}$ \\
$\mathbf{8}$ & $\begin{array}{l}\text { Complete } \\
\text { stricture }\end{array}$ & $\begin{array}{l}\text { Soft tissue } \\
\text { stricture site }\end{array}$ \\
$\mathbf{9}$ & $\begin{array}{l}\text { Complete } \\
\text { stricture }\end{array}$ & $\begin{array}{l}\text { Soft tissue at } \\
\text { stricture site }\end{array}$ \\
$\mathbf{1 0}$ & $\begin{array}{l}\text { Narrowed } \\
\text { urethra }\end{array}$ & $\begin{array}{l}\text { Periurethral } \\
\text { Haematoma }\end{array}$ \\
$\mathbf{1 1}$ & $\begin{array}{l}\text { Narrowed } \\
\text { urethra }\end{array}$ & Periurethral abscess \\
& $\begin{array}{l}\text { Dilated } \\
\text { post. } \\
\text { urethra } \\
\text { with abrupt } \\
\text { cut up }\end{array}$ & $\begin{array}{l}\text { Dilated posterior } \\
\text { urethra with fold of } \\
\text { membrane }\end{array}$ \\
\hline
\end{tabular}

Two cases of urethral calculus seen on RGU were correctly identified on sonourethrography

Two cases of narrowing at bulbar urethra /Posterior part of penile Urethra on RGU showed periurethral abscess $(17.4 \times 10.8 \mathrm{~mm})$ in one case and periurethral haematoma in other case on sonourethrography. Though the lesions of urethra identified on RGU, but it is due to periurethral abnormality is identified only on sonourethrography.

All the cases of urethral lesion (12 normal and 15 abnormal) on RGU were correctly identified on Sonourethrography (Sensitivity $100 \%$ ), but with additional information as mucosal irregularity in two normal cases. periurethral causes for urethral abnormality were completely identified on sonourethrography while they were not identified on RGU. 


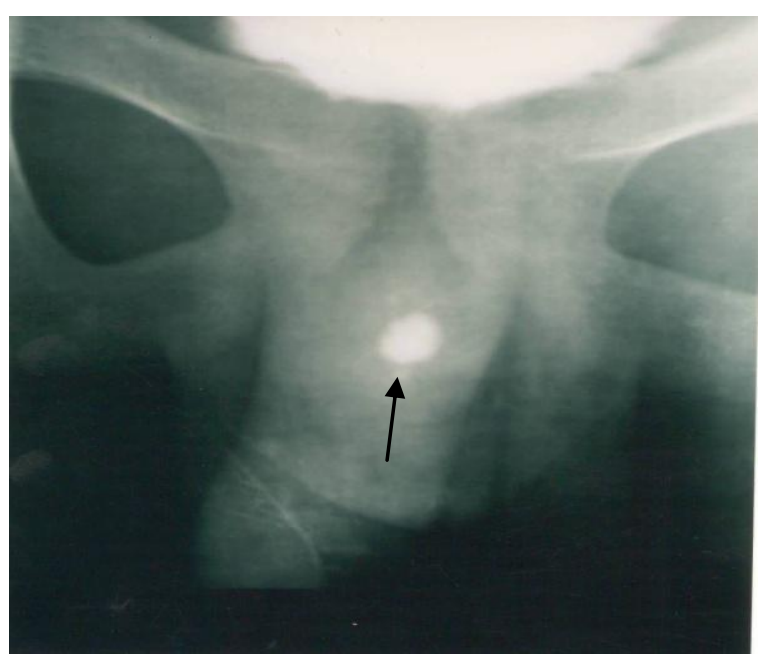

Fig 3: MCU where patient could not void on table showing radiopaque calculus in penile urethra (arrow).

\section{Discussion}

Retrograde and ante grade urethrography still remains the most common investigation in imaging evaluation of urethra. Sonourethrography is able to assess both the urethral and periurethral tissue. Posterior urethral valves (PUV) are one of the common congenital anomalies of the urethra in males. In the present study one case of PUV was present which was diagnosed both on MCU and sonourethrography. . Infectious urethritis present clinically as dysuria and urethral discharge and radiographic findings of acute disease include irregularity of the lumen with filling defects due to sloughed mucosa. Features of chronic disease include luminal irregularity, stricture formation commonly in bulbar urethra. In the present study none of the acute cases were included, because of risk of retrograde spread of the infection to urinary bladder and kidney. Radiographic retrograde urethrography has traditionally been the gold standard for imaging the anterior urethra, sonourethrography has proved to be precise and effective for evaluating urethral strictures. Measurement of the stricture length in the bulbar urethra is more accurate than conventional retrograde urethrography while spongiofibrosis is manifested by lack of urethral distensibility during retrograde installation of saline solution. ${ }^{1}$ Morey AF et al reported that Sonourethrography has major influence on selection of therapy in patients with bulbar strictures of intermediate length. ${ }^{2}$ In 1995 Arda K et al. reported that the sonourethrography and radiographic urethrography can substitute each other in determining the stricture length of anterior urethra and both of these methods are used in combination, it will be more sensitive. ${ }^{3}$

Sonourethrography is noninvasive that can provide valuable information about the urethral lumen and the urethral wall. ${ }^{4}$ Gupta $S$ et al. in 1993 carried out sonourethrographic evaluation of anterior urethral stricture in 30 patients and correlated the result with radiographic urethrography. ${ }^{5}$ Their result showed that sonourethrography was an accurate predictor of stricture length while retrograde urethrography underestimated the length in most of the cases. Further they pointed out that sonourethrography was unsatisfactory in evaluation of membranous stricture. ${ }^{5}$ Included seven cases in the present study showed stricture in anterior (penile and bulbar) urethra were chronic cases of urethritis. In the present study all the cases of stricture were diagnose correctly on RGU. Sonourethrography provided more accurate measurement of stricture length as presented in table II.

Degree of luminal narrowing and periurethral abnormalities was better depicted on sonourethrography. Two cases of urethral narrowing identified on RGU 
were due to periurethral lesion as recognized on sonourethrography. One case was due to periurethral abscess and other was due to periurethral hematoma in a patient with history of urethral injury. Two cases of complete stricture on RGU, sonourethrography identified soft tissue density lesion causing complete cut up of urethra and urethra could not be traced further.. Sonourethrography is better for evaluating mucosal and periurethral abnormalities hence it should be preferred whenever periurethral abnormality is suspected. Three normal urethra on RGU, showed mucosal Flap (bulge) in one and mucosal irregularity in two on sonourethrography. Derschess W in 1992 described sonourethrography as painless quick and cheap procedure and has nearly the same diagnostic value as conventional method. ${ }^{8}$

In the present study sonourethrography showed sensitivity of $100 \%$ in identifying the urethral abnormalities. Traumatic injury most commonly involves membranous urethra, where it is relatively fixed within urogenital diaphragm and subjected to shearing forces. Urethral injury should be suspected and evaluated for in patients with pelvic fractures associated with haematuria. In present study one case of haematuria following RTA was included. Urethral injury occurs in $10 \%$ cases of pelvic fractures and are classified into three types. ${ }^{13}$

Type I stretched urethra secondary to haematoma, no tear, type II urethral tear above urogenital diaphragm, type III urethral tear below the urogenital diaphragm. In the present study none of the cases of urethral tear were present. Urethral calculus usually is due to bladder calculus migrated down in to urethra. In the present study two case of urethral calculus were correctly identified on both RGU and sonourethrography. Two cases of urethral narrowing on RGU were identified as periurethral abscess in one and haematoma in other on sonourethrography.

\section{Conclusion}

Radiographic urethrography (MCU/RGU) though gold standard in evaluating urethral abnormalities, Sonourethrography is eqully accurate (sensitivity 100\%) in the diagnosis of urethral lesions. Sonourethrography has advantage in better evaluating mucosal and periurethral abnormalities as cause of urethral lesions. Further Sonourethrography is more accurate in measurement of stricture length and it is cheap, safe and does not provide any kind of radiation to the patient in gonadal areas.

\section{Reference}

1. Morey AF, McAninch JW. Sonographic staging of anterior urethral stricture. J Urol. 2000;163(4):1070-5.

2. Morey AF, McAninch JW. Role of preoperative sonourethrography in bulbar reconstruction.J Urol. 1997;158(4):13769.

3. Arda K, Basar M, Deniz E, et al. Sonourethrography in anterior urethral stricture:comparison to radiographic urethrography. Arch Italurol Androl. 1995;67(4):249-54.

4. Montanari E, Deiana G, Cogni M, et al. Validity of superficial echography in the 
study of urethral pathology. Arch Ital Urol Androl.1994;66(4 suppl):113-7.

5. Gupta S, Majumdar B, Tiwari A, et al. Sonourethrography in the evaluation of anterior urethral strictures:correlation with Radiographic urethrography. J Clin Ultrasound. 1993;21(4):231-9.

6. Fernandez A, Ramirez Esteban A, Gil Fbra J, et al. Normal echographic anatomy of the penis and the perineum. Arch Esp Urol.1992;45(7):695-8.

7. Moncada IJ, Garcia LE, Verdu TF, et al. Urethralsonography in the diagnosis and preoperative evaluation of urethral stenosis. Actas Urol Esp.1992;16(6):4626.

8. Derschum W: Is ultrasound imaging of the urethra an alternative to radiologic urethral imaging and urethroscopy? Urologe A.1992;31(3):177-8.
9. Vapnek JM, Hricak H, Carrol PR. Recent advances in imaging studies for staging of penile and urethral carcinoma. Urol Clin North Am.1992;19(2):257-66.

10. Eapuela OR, Abad MF, Gimeno MA, et al. Integral urologic echography: retrograde sonourethrography. Arch Esp Urol.1992;45(3):237-42.

11. Klosterman PW, Laing FC, McAninch JW. Sonourethrography in the evaluation of urethral stricture disease. Urol Clin North Am.1989;16(4):791-7.

12. McAninch JW, Laing FC, Jeffrey RBJ. Sonourethrography in the evaluation of urethral strictures: a preliminary report. J Urol.1988;139(2):294-7.

13. Colapinto V, McCallum RW. Injury to male posterior urethra in fractured pelvis: a new classification. J Urol.1977;118:575-80. 